\title{
Probing strongly hybridized nuclear-electronic states in a model quantum ferromagnet
}

\author{
I. Kovacevic,,${ }^{1, *}$ P. Babkevich,,${ }^{1, \dagger}$ M. Jeong (정 민 기), ${ }^{1, \ddagger}$ J. O. Piatek, ${ }^{1}$ G. Boero, ${ }^{2}$ and H. M. Rønnow ${ }^{1}$ \\ ${ }^{1}$ Laboratory for Quantum Magnetism, Institute of Physics, Ecole Polytechnique Féderale de Lausanne (EPFL), \\ CH-1015 Lausanne, Switzerland \\ ${ }^{2}$ Microsystems Laboratory, Ecole Polytechnique Féderale de Lausanne (EPFL), CH-1015 Lausanne, Switzerland \\ (Received 9 September 2016; revised manuscript received 11 November 2016; published 28 December 2016)
}

\begin{abstract}
We present direct local-probe evidence for strongly hybridized nuclear-electronic spin states of an Ising ferromagnet $\mathrm{LiHoF}_{4}$ in a transverse magnetic field. The nuclear-electronic states are addressed via a magnetic resonance in the $\mathrm{GHz}$ frequency range using coplanar resonators and a vector network analyzer. The magnetic resonance spectrum is successfully traced over the entire field-temperature phase diagram, which is remarkably well reproduced by mean-field calculations. Our method can be directly applied to a broad class of materials containing rare-earth ions for probing the substantially mixed nature of the nuclear and electronic moments.
\end{abstract}

DOI: 10.1103/PhysRevB.94.214433

The compound $\mathrm{LiHoF}_{4}$ is widely regarded as a prototypical system realizing the transverse-field Ising model [1]. The ground state in zero field is ferromagnetically ordered, while applying a relatively small transverse field induces a zerotemperature quantum phase transition at $H_{c}=4.95 \mathrm{~T}$ into a quantum paramagnet [2], as shown in Fig. 1. Meanwhile, the hyperfine coupling strength of a $\mathrm{Ho}^{3+}$ ion is exceptionally large, with a coupling constant $A=39(1) \mathrm{mK}[3,4]$. The resulting strong hybridization between the electronic and nuclear magnetic moments [5] leads to two dramatic effects close to the quantum critical point: (i) significant modification of the low-temperature magnetic phase boundary (see Fig. 1) [2]; and (ii) incomplete mode softening of the low-energy electronic excitations at the critical point [6]. Therefore, this system provides a rare opportunity to explore the quantum phase transition of a magnet coupled to a nuclear spin bath [2,6-8].

The impact of strong hybridization has also been highlighted for magnetic-ion diluted insulators, such as $\mathrm{LiYF}_{4}: \mathrm{Ho}^{3+}$, using magnetic resonance $[9,10]$. A similar line of effort has achieved more recently single-molecule magnetic resonance with a rare-earth ion [11]. Furthermore, strong hybridization is of great interest in quantum information science [12-14]. As much as these examples focus on the single-ion limit, the other limiting case of many-body systems, such as $\mathrm{LiHoF}_{4}$, provides a very different and complementary perspective. While in the long-range-ordered state the hybridization is suppressed, an applied transverse field introduces quantum fluctuations enhancing the hybridization towards $H_{c}$.

However, probing directly the strongly hybridized states in $\mathrm{LiHoF}_{4}$ using spectroscopic methods, at the lowest-energy scale, has so far been restricted to the thermal paramagnetic phase in the single-ion limit. The involved energy scale is too low to be resolved by neutron scattering [6,7]. Magnetic resonance on ${ }^{165}$ Ho nuclei would provide a direct way of probing the hybridized nuclear-electronic states. However, the resonance in the ordered phase is expected around the

\footnotetext{
*ivankowacevic@gmail.com

†peter.babkevich@gmail.com

${ }^{\ddagger}$ minki.jeong@gmail.com
}

frequency of $4.5 \mathrm{GHz}$ in zero field, which does not fall into the operating frequencies of conventional nuclear magnetic resonance (NMR) nor electron spin resonance (ESR) instrumentation. Some studies have reported a hyperfine structure in ESR [3,15], but all in the paramagnetic regime above the ordering temperature $T_{c}=1.53 \mathrm{~K}$ [2]. To date, microscopic evidence for the realization of the unique nuclear-electronic Ising model $[16,17]$ is absent.

Here, we demonstrate experimentally nuclear-electronic magnetic resonance in $\mathrm{LiHoF}_{4}$ using coplanar microwave resonators and a vector network analyzer (VNA). We successfully trace the temperature and field evolution of the spectrum over the entire phase diagram, and show that it is remarkably well reproduced by a mean-field calculation with parameters set by independent spectroscopic measurements $[3,4,7,8]$.

We begin with a description of our experimental setup shown in Fig. 2. A series of microwave coplanar resonators with different fundamental frequencies from 1.7 to $5.6 \mathrm{GHz}$ were prepared. The impedance of the resonator is matched to the rest of the system by optimizing the gap size between the conductors. The oscillating magnetic field $\mathbf{B}(t)$ generated at the sample position is parallel to the surface. A cube shaped sample of $2 \times 2 \times 2 \mathrm{~mm}^{3}$ was placed at the center of the active strip, with a submillimeter gap in between to avoid unwanted heating. The measurement geometry was chosen such that the applied magnetic field $\mathbf{B}_{0}$ is along the crystallographic $b$ axis of the tetragonal scheelite structure, and $\mathbf{B}(t)$ is perpendicular to both $\mathbf{B}_{0}$ and the $c$ axis to satisfy the magnetic resonance condition. We measured the $S_{11}$ parameter, which is defined as the ratio of the reflected to the input power, using a VNA which is connected through a low-loss cryogenic coaxial cable to the coplanar resonator. The coaxial cable was thermally anchored at each stage of the dilution refrigerator including the $1 \mathrm{~K}$ pot, still, and mixing chamber to ensure thermalization. The sample thermometer was located only $5 \mathrm{~mm}$ away from the sample which was thermally anchored to the mixing chamber. With an input power of $-16 \mathrm{dBm}$ applied by the network analyzer, the sample base temperature was $0.15 \mathrm{~K}$ to within $0.01 \mathrm{~K}$.

To guide and interpret our experimental investigation, we perform a model calculation using a mean-field approximation. The full Hamiltonian has been well characterized through a 


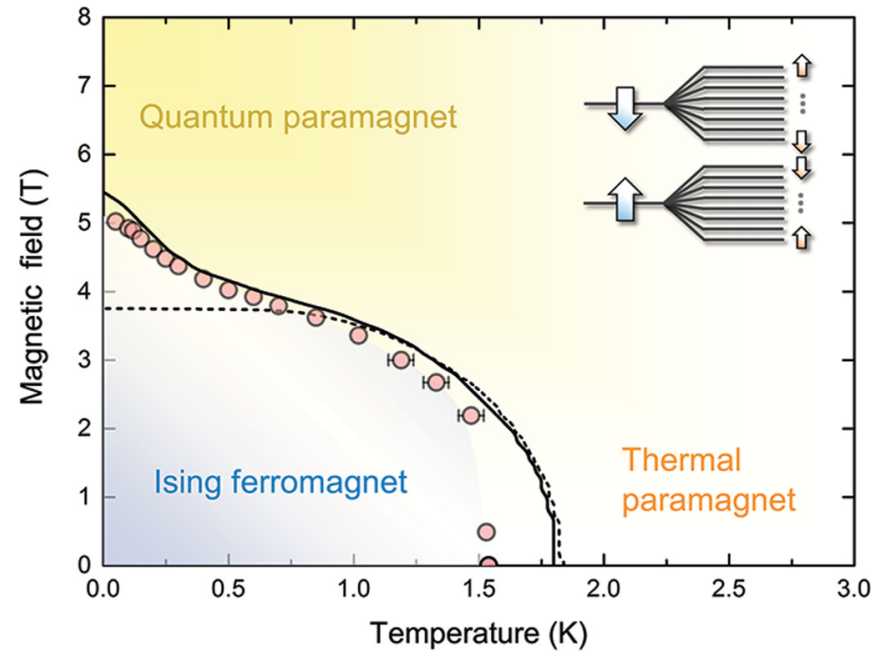

FIG. 1. Phase diagram of $\mathrm{LiHoF}_{4}$ in a transverse magnetic field with the experimental data taken from Ref. [2]. The solid line represents a mean-field calculation following Ref. [7], taking into account a strong hyperfine interaction, while the dashed line is calculated without a hyperfine interaction. The inset shows schematic energy levels for the Ising spins in the ordered phase (left) and its modification by hyperfine interactions with the nuclear spins (right).

number of different experiments $[3,7]$ and is given by

$$
\begin{aligned}
\mathcal{H}= & \sum_{i}\left[\mathcal{H}_{\mathrm{CF}}\left(\hat{\mathbf{J}}_{i}\right)+A \hat{\mathbf{J}}_{i} \cdot \hat{\mathbf{I}}_{i}-g_{L} \mu_{\mathrm{B}} \hat{\mathbf{J}}_{i} \cdot \mathbf{B}_{\mathbf{0}}\right] \\
& -\frac{1}{2} \sum_{i j} \sum_{\alpha \beta} \mathcal{J}_{\mathrm{D}} D_{\alpha \beta} \hat{J}_{i \alpha} \hat{J}_{j \beta}-\frac{1}{2} \sum_{\langle i j\rangle} \mathcal{J}_{\mathrm{ex}} \hat{\mathbf{J}}_{i} \cdot \hat{\mathbf{J}}_{j},
\end{aligned}
$$

where $\hat{\mathbf{J}}_{i}(J=8)$ and $\hat{\mathbf{I}}_{i}(I=7 / 2)$ are the electronic and nuclear angular momentum operators at site $i$, the dipolar coupling constant $\mathcal{J}_{\mathrm{D}}=n\left(g_{L} \mu_{B}\right)^{2}=13.5 \mathrm{mK}, D_{\alpha \beta}$ is the dimensionless coupling parameter for the dipole-dipole interaction [18], and the negligible exchange constant $J_{\mathrm{ex}}=$ $-1.2 \mathrm{mK}$. The nuclear Zeeman and quadrupole interactions are assumed to be negligible [9]. The crystal field interaction $\mathcal{H}_{\mathrm{CF}}$ with the surrounding ions splits the electronic states, resulting in a ground state which is a non-Kramers doublet with a strong Ising-like anisotropy and the first excited state $11 \mathrm{~K}$ above. In the ordered state, dipolar coupling lifts the ground-state degeneracy, resulting in pseudospins up and down which we label as $|\uparrow\rangle$ and $|\downarrow\rangle$ states. Each state is further split into eight

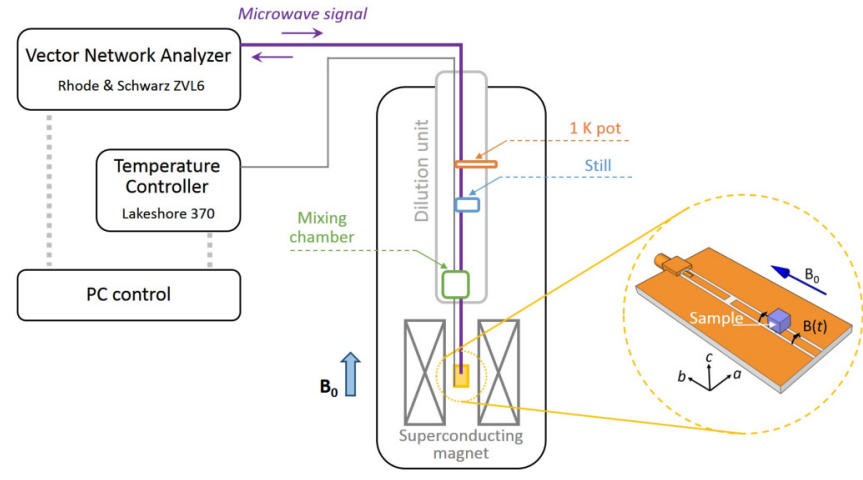

FIG. 2. Schematic drawing of the setup showing the sample mounted on top of the microwave coplanar resonator inside the vertical field magnet.

nuclear-electronic states by the hyperfine interaction [Fig. 1(a), inset].

The total Hamiltonian can be diagonalized in the basis of $(2 J+1) \times(2 I+1)=136$ nuclear-electronic $|\alpha\rangle=$ $\left|m_{J}, m_{I}\right\rangle$ states. The evolution of the lowest states with the applied transverse field is shown in Fig. 3(a). The energy level difference between consecutive states $\Delta E$ changes dramatically with the field, as illustrated in Fig. 3(b). In the first approximation, $\Delta E$ is proportional to $A|\langle\mathbf{J}\rangle|$, where $|\langle\mathbf{J}\rangle|$ is the magnitude of the total angular momentum, hence $\Delta E$ decreases with the field and reaches a minimum at $H_{c}$. The diagram shown in Fig. 3(b) allows us to predict at which field the magnetic resonance occurs for a given frequency.

Experimentally, we observed magnetic transitions between the adjacent nuclear-electronic levels through resonant absorption of continuous microwaves by the sample on a coplanar resonator. Figure 3(c) presents a typical frequency-field map at $0.3 \mathrm{~K}$ of the $S_{11}$ parameter using a resonator with the unloaded frequency of $3.4 \mathrm{GHz}$. The map shows a clear anomaly around 3.6 T, indicative of magnetic resonance. This field value indeed agrees with the one predicted by mean-field calculations, which can be seen in Fig. 3(b) by taking an intersect of the blue dashed line for $3.4 \mathrm{GHz}$ with the solid lines for the energy level difference.

For an in-depth comparison between experiments and calculations, we proceed to directly calculate the imaginary part of the frequency-dependent susceptibility $\chi^{\prime \prime}(f)$ which is responsible for magnetic resonance absorption $[19,20]$. The calculations were performed within the linear-response framework [18] using the mean-field wave functions $|\alpha\rangle$ and $\left|\alpha^{\prime}\right\rangle$,

$$
\chi^{\prime \prime}(f)=\sum_{\alpha \alpha^{\prime}} \frac{\left\langle\alpha\left|\left(g_{L} \mu_{B} \hat{J}_{y}+g_{N} \mu_{N} \hat{I}_{y}\right)\right| \alpha^{\prime}\right\rangle\left\langle\alpha^{\prime}\left|\left(g_{L} \mu_{B} \hat{J}_{y}+g_{N} \mu_{N} \hat{I}_{y}\right)\right| \alpha\right\rangle}{\left(E_{\alpha^{\prime}}-E_{\alpha}-h f\right)^{2}+\Gamma_{\alpha^{\prime} \alpha}^{2}} \Gamma_{\alpha^{\prime} \alpha}\left(n_{\alpha}-n_{\alpha^{\prime}}\right)+\chi^{\prime}(0),
$$

where $E_{\alpha}$ is the energy of the hybridized nuclear-electronic eigenstates in the presence of the mean field, $n_{\alpha}=$ $\exp \left(-\beta E_{\alpha}\right) / Z$ is the thermal population factor, and $Z=$ $\sum_{\alpha^{\prime}} \exp \left(-\beta E_{\alpha^{\prime}}\right)$ is the partition function. The subscript $y$ refers to the oscillating field direction. The lifetime in the linear-response calculation of the states is assumed to be independent of field and temperature, and was fixed to $40 \mathrm{~ns}$, corresponding to a damping of $\Gamma_{\alpha^{\prime} \alpha}=0.17 \mathrm{GHz}$, which 
(a)

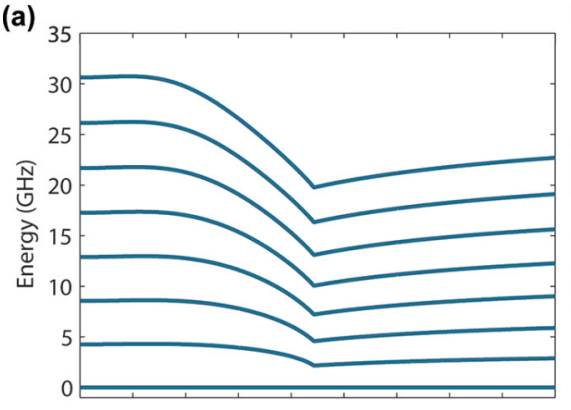

(b)

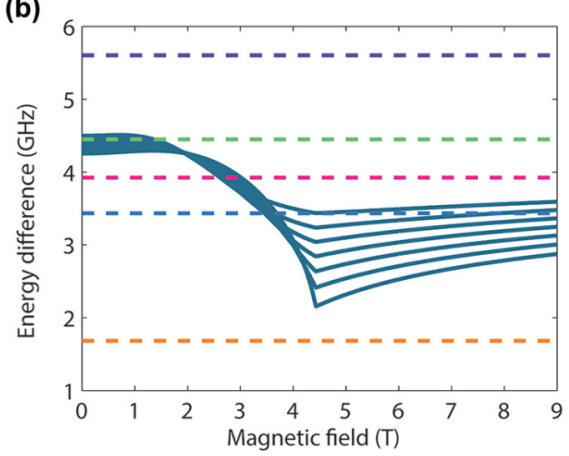

(c)

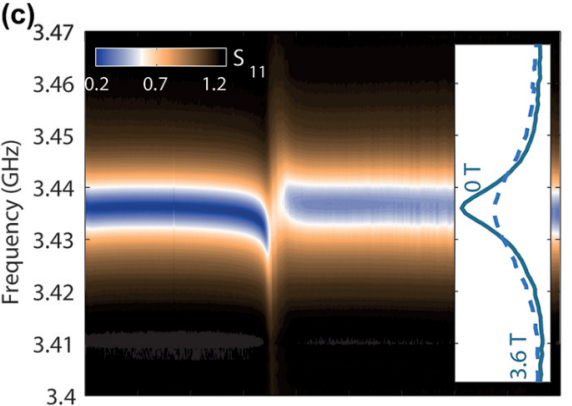

(d)

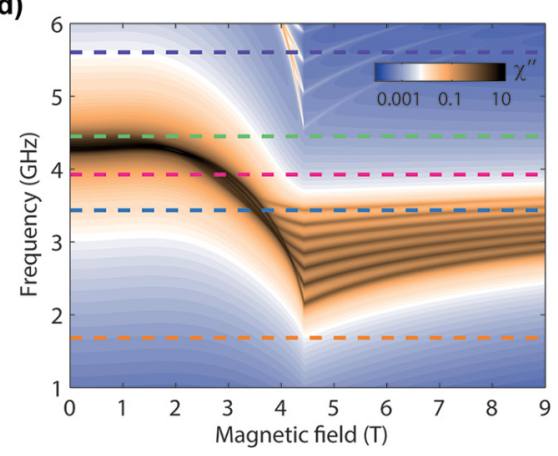

(e)

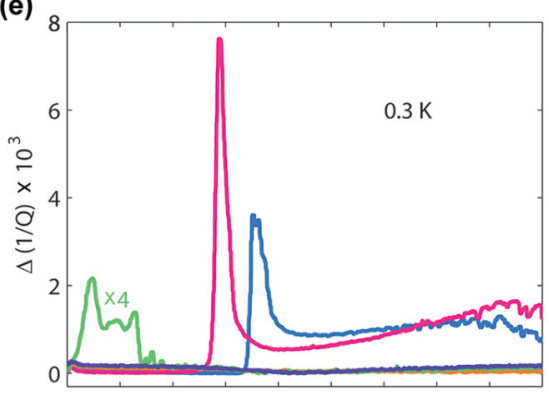

(f)

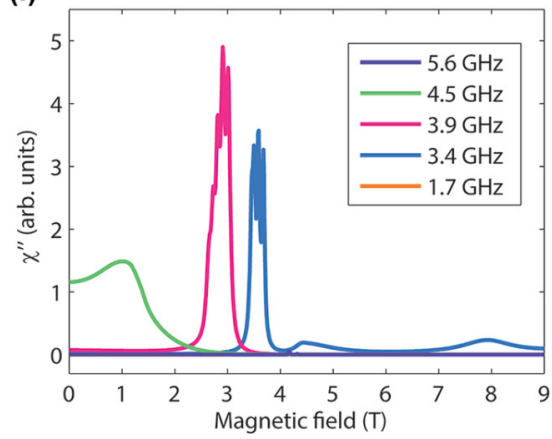

FIG. 3. (a) Calculated energy levels for the nuclear-electronic ground state in the mean-field approximation as a function of transverse magnetic field, and (b) the field evolution of the corresponding energy difference between the adjacent levels. The calculations were performed for $0.3 \mathrm{~K}$ to compare with the experiments. Magnetic resonance would occur when the excitation frequency (dashed lines) intersects with the energy difference. (c) Frequency-field map of the experimental $S_{11}$ parameter, where the anomaly around $3.6 \mathrm{~T}$ corresponds to the expected resonance field for the frequency of $3.4 \mathrm{GHz}$. The inset shows constant-field cuts of the $S_{11}$ map at (dashed) and away (solid) from the resonance. (d) Frequency-field map of the calculated $\chi^{\prime \prime}$ intensity. (e) Experimental magnetic resonance spectra obtained for several different frequencies, and (f) the calculated $\chi^{\prime \prime}$ for the corresponding frequencies.

provided the best match to our data. The lifetime broadening may result from direct or indirect contributions from the electronic dipolar and exchange or nuclear dipolar couplings [20], which we leave for future study. We note that the contribution to susceptibility from electronic moments $\hat{J}_{y}$ is 500 times larger than the contribution from nuclear moments $\hat{I}_{y}$. Therefore, despite the predominantly nuclear-spin nature of the $|\uparrow\rangle$ levels, the response we measure comes mainly from the electrons. This gives a tremendous enhancement of the signal from the nuclear states amplified by electronic moments. Figure 3(d) presents the calculated frequency-field map of $\chi^{\prime \prime}$ intensity at $0.3 \mathrm{~K}$, which shows a drastic change upon approaching $H_{c}$ from below. Resonant absorption is expected from our calculations to be in the $2-4.5 \mathrm{GHz}$ bandwidth.

The absorptive part of the susceptibility is experimentally estimated as $\chi^{\prime \prime} \propto \Delta(1 / Q)$ [19], where the quality factor $Q$ is defined as the loaded frequency divided by the full width at half maximum in the absorption profile in frequency, as shown in the inset of Fig. 3(c). In Fig. 3(e) we show the experimental magnetic resonance spectra at $0.3 \mathrm{~K}$ for several different frequencies by plotting $\Delta(1 / Q)=1 / Q-b$, where $b$ is a uniform background, which can be compared to the calculated spectra at $0.3 \mathrm{~K}$ in Fig. 3(f). Both calculations and measurements at frequencies of 3.4 and $3.9 \mathrm{GHz}$ show resonant peaks around 3.6 and $3.0 \mathrm{~T}$, respectively. Conversely, no resonance features are visible for the frequency of $1.7 \mathrm{GHz}$ in both calculations and experiments. The predicted transitions between second-nearest-neighboring levels at $5.6 \mathrm{GHz}$ is too weak to be observed experimentally. The calculated spectrum for $4.5 \mathrm{GHz}$ appears as a broad hump at fields below $2 \mathrm{~T}$, which can be expected from Fig. 3(d), where the frequency line cuts along the strongest $\chi^{\prime \prime}$ intensity. For a better comparison, the $A$ value was slightly reduced by $3 \%$, which is nearly within the uncertainty from the reported one [3]. In principle, the uncertainty in the crystal field parameters can influence our calculations [8]. Nevertheless, excellent agreement with the experiments is remarkable considering that the model is essentially parameter free. Some minor discrepancies such as the fine structure in the $4.5 \mathrm{GHz}$ experimental spectrum are likely due to the fixed lifetime of all levels in our model. However, since the modes around $4.5 \mathrm{GHz}$ lie very close in the relevant field range, that structure would depend critically on the tiny variations of parameters. We therefore consider it more prudent to use a constant damping. The high-field tails in 3.4 and $3.9 \mathrm{GHz}$ spectra are possibly due to the neglected effects of fluctuations.

Furthermore, we investigate the temperature evolution of the spectrum for $3.4 \mathrm{GHz}$ from 0.15 to $2.5 \mathrm{~K}$, as shown in Fig. 4(a). At base temperature a resonance peak appears around 3.7 T, which on warming decreases in amplitude and shifts to lower fields. The former is due to redistribution of the thermal population of states at higher temperatures. The latter reflects the decreasing size of the ordered electronic moment with increasing temperature, sensed by the nuclei through the hyperfine interactions. In Fig. 4(b) we track the resonance field as a function of temperature. Our measurements are shown to 
(a)

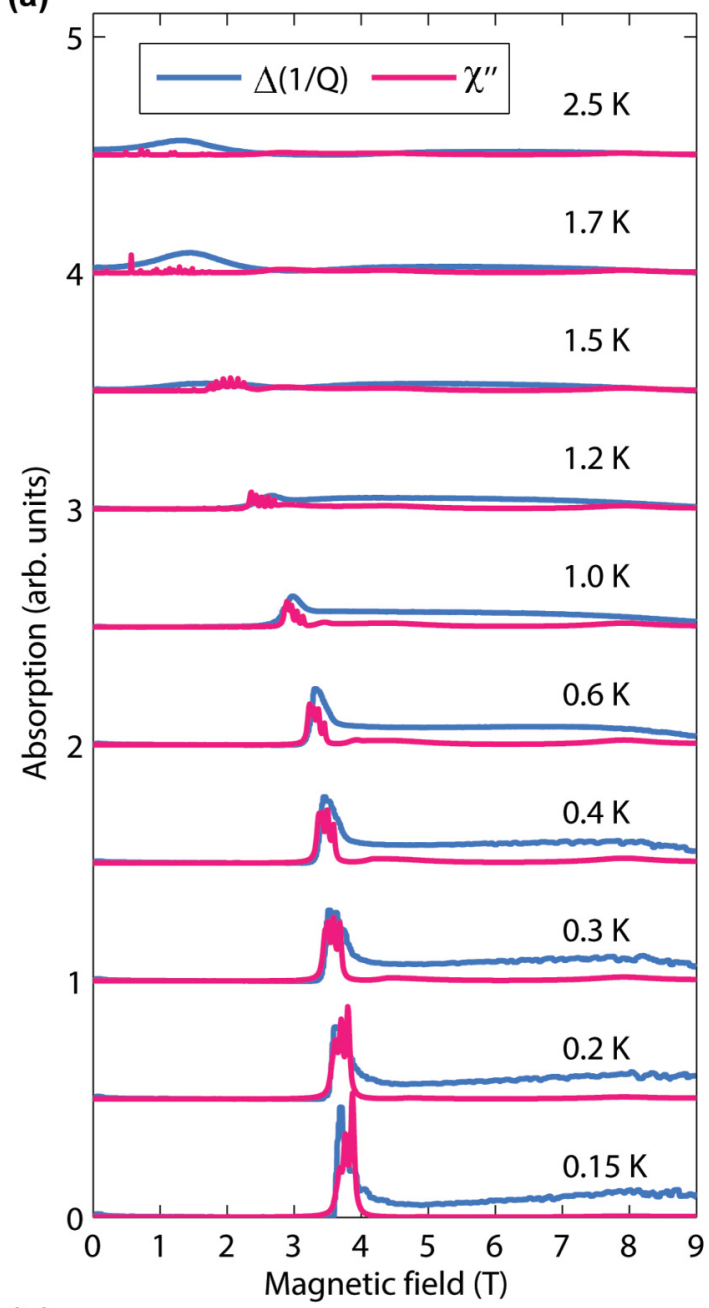

(b)

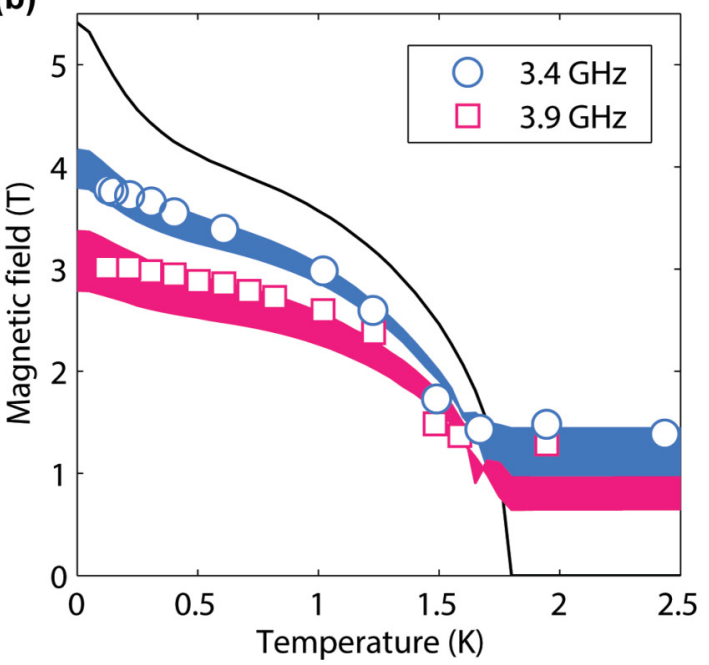

FIG. 4. (a) Temperature evolution of the spectra from 0.15 to $2.5 \mathrm{~K}$, from experiments (blue) and calculations (red), using an excitation frequency of $3.4 \mathrm{GHz}$. (b) The resonance field as a function of temperature for two different frequencies. The colored bands are calculations using hyperfine constant in the range of $\pm 3 \%$ from the value used in Fig. 3(f). The black line reproduces the calculated phase boundary.

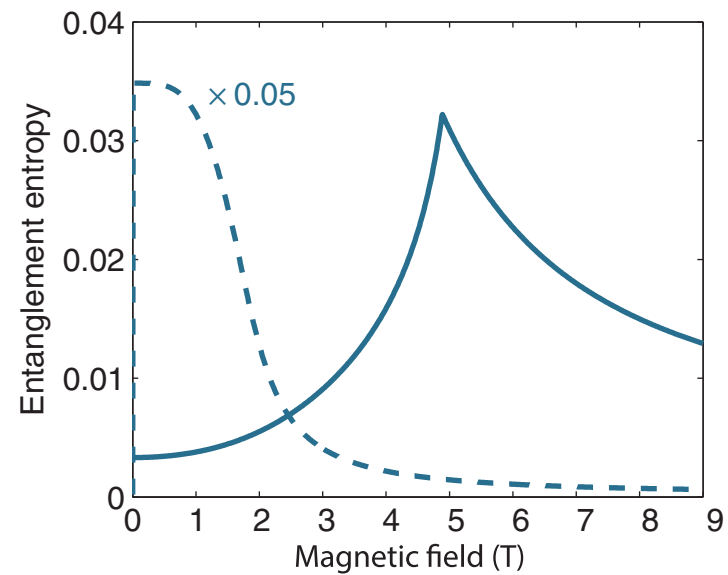

FIG. 5. Entanglement entropy calculated for the ground state of $\mathrm{LiHoF}_{4}$ as a function of transverse magnetic field (solid line). The dashed line is the calculation without dipolar interactions.

be very sensitive to small variations of the hyperfine coupling as depicted by the bands.

As shown in Figs. 3 and 4, all the salient features of the experimental results are well reproduced by the model calculations, thereby validating the transverse-field nuclearelectronic Ising model $[16,17]$. The excellent description of the experimental results by our model implies that the probed states have a strongly hybridized character of both nuclear and electronic degrees of freedom. While this has been only hinted at by previous bulk measurements [2] and neutron spectroscopy [6], here we show directly the transitions between the strongly hybridized nuclear-electronic states. Likewise, the presented magnetic resonance should be distinguished from conventional NMR and ESR where the electronic and nuclear moments are approximated to product states [19-21].

To highlight the qualitative difference in the hybridized states between those in the many-body system and in the single-ion limit, we calculate the ground-state entanglement entropy $[22,23]$ between the electronic and nuclear moments as a measure of the hybridization. We employ the Schmidt decomposition of the mean-field wave function $|\Psi\rangle=$ $\sum_{n} c_{n}\left|m_{J}\right\rangle \otimes\left|m_{I}\right\rangle$, where $c_{n} \geqslant 0$ and $\sum_{n} c_{n}^{2}=1$, where the entanglement entropy is given by the von Neumann entropy $S=-\sum_{n}\left|c_{n}\right|^{2} \ln \left|c_{n}\right|^{2}$. The calculated entropy in the absence of dipolar interactions decreases smoothly with a transverse field (Fig. 5), in agreement with those reported by Ref. [17]. However, by turning on dipolar coupling the model produces a cusplike peak at $H_{c}$, that is, the hybridization in the ordered state of $\mathrm{LiHoF}_{4}$ increases with the applied field until it reaches a peak at the critical point. The field essentially mixes the higher excited states into the ground state, thereby enhancing the hybridization. The increasingly larger field, $H>H_{c}$, magnetizes the electronic and nuclear moments along the field direction such that the ground state approaches a product state.

To summarize, we have demonstrated Ho nuclear-electronic magnetic resonance of $\mathrm{LiHoF}_{4}$ in a transverse magnetic field over the entire field-temperature phase diagram. The spectral evolution is remarkably well reproduced by mean-field calculations, validating the transverse-field nuclear-electronic Ising model. Taking advantage of the well-characterized model 
nature of $\mathrm{LiHoF}_{4}$, we have successfully probed the strongly hybridized states and their evolution in the long-range-ordered state. Our experimental scheme will find direct applications not only in the $\mathrm{Li} \mathrm{FF}_{4}$ ( $R=$ rare earth) family $[8,24,25]$, but also other $R$ containing compounds including spin glass $[16,17,26-28]$ and spin ice $[29,30]$.

We are grateful to M. Graf, S. S. Kim, and P. Jorba Cabre for their contribution in building the experimental setup at the initial stage, and B. Dalla Piazza for sharing his insight into the mean-field and linear-response theory. We also thank J. Jensen, A. Feofanov, and D. Yoon for helpful discussions. M.J. is grateful for support by the European Commission through Marie Skłodowska-Curie Action COFUND (EPFL Fellows). This work was supported by the Swiss National Science Foundation, the MPBH network and European Research Council grant CONQUEST.

I.K., P.B., and M.J. contributed equally to this work.
[1] S. Sachdev, Quantum Phase Transitions (Cambridge University Press, Cambridge, UK, 1999).

[2] D. Bitko, T. F. Rosenbaum, and G. Aeppli, Phys. Rev. Lett. 77, 940 (1996)

[3] J. Magariñno, J. Tuchendler, P. Beauvillain, and I. Laursen, Phys. Rev. B 21, 18 (1980).

[4] G. Mennenga, L. de Jongh, and W. Huiskamp, J. Magn. Magn. Mater. 44, 59 (1984).

[5] One may consider the hybridization strong once the hyperfine coupling strength is comparable to the Zeeman energy scale; for instance, see Ref. [13].

[6] H. M. Rønnow, R. Parthasarathy, J. Jensen, G. Aeppli, T. F. Rosenbaum, and D. F. McMorrow, Science 308, 389 (2005).

[7] H. M. Rønnow, J. Jensen, R. Parthasarathy, G. Aeppli, T. F. Rosenbaum, D. F. McMorrow, and C. Kraemer, Phys. Rev. B 75, 054426 (2007).

[8] P. Babkevich, A. Finco, M. Jeong, B. Dalla Piazza, I. Kovacevic, G. Klughertz, K. W. Krämer, C. Kraemer, D. T. Adroja, E. Goremychkin, T. Unruh, T. Strässle, A. Di Lieto, J. Jensen, and H. M. Rønnow, Phys. Rev. B 92, 144422 (2015).

[9] R. Giraud, W. Wernsdorfer, A. M. Tkachuk, D. Mailly, and B. Barbara, Phys. Rev. Lett. 87, 057203 (2001).

[10] R. Giraud, A. M. Tkachuk, and B. Barbara, Phys. Rev. Lett. 91, 257204 (2003).

[11] S. Müllegger, S. Tebi, A. K. Das, W. Schöfberger, F. Faschinger, and R. Koch, Phys. Rev. Lett. 113, 133001 (2014).

[12] G. W. Morley, M. Warner, A. M. Stoneham, P. T. Greenland, J. van Tol, C. W. Kay, and G. Aeppli, Nat. Mater. 9, 725 (2010).

[13] G. W. Morley, P. Lueders, M. H. Mohammady, S. J. Balian, G. Aeppli, C. W. Kay, W. M. Witzel, G. Jeschke, and T. S. Monteiro, Nat. Mater. 12, 103 (2013).

[14] M. Shiddiq, D. Komijani, Y. Duan, A. Gaita-Ariño, E. Coronado, and S. Hill, Nature (London) 531, 348 (2016).

[15] P. Janssen, I. De Wolf, and I. Laursen, J. Phys. Chem. Solids 46, 1387 (1985).
[16] M. Schechter and P. C. E. Stamp, Phys. Rev. Lett. 95, 267208 (2005).

[17] M. Schechter and P. C. E. Stamp, Phys. Rev. B 78, 054438 (2008).

[18] J. Jensen and A. R. Mackintosh, Rare Earth Magnetism (Clarendon, Oxford, UK, 1991).

[19] B. Cowan, Nuclear Magnetic Resonance and Relaxation (Cambridge University Press, Cambridge, UK, 2005).

[20] A. Abragam and M. Goldman, Nuclear Magnetism (Clarendon, Oxford, UK, 1982).

[21] A. Abragam and B. Bleaney, Electron Paramagnetic Resonance of Transition Ions (Oxford University Press, Oxford, UK, 2012).

[22] M. A. Nielsen and I. L. Chuang, Quantum Computation and Quantum Information (Cambridge University Press, Cambridge, UK, 2010).

[23] C. H. Bennett, H. J. Bernstein, S. Popescu, and B. Schumacher, Phys. Rev. A 53, 2046 (1996).

[24] C. Kraemer, N. Nikseresht, J. O. Piatek, N. Tsyrulin, B. Dalla Piazza, K. Kiefer, B. Klemke, T. F. Rosenbaum, G. Aeppli, C. Gannarelli et al., Science 336, 1416 (2012).

[25] P. Babkevich, M. Jeong, Y. Matsumoto, I. Kovacevic, A. Finco, R. Toft-Petersen, C. Ritter, M. Månsson, S. Nakatsuji, and H. M. Rønnow, Phys. Rev. Lett. 116, 197202 (2016).

[26] D. Silevitch, D. Bitko, J. Brooke, S. Ghosh, G. Aeppli, and T. Rosenbaum, Nature (London) 448, 567 (2007).

[27] C. Ancona-Torres, D. M. Silevitch, G. Aeppli, and T. F. Rosenbaum, Phys. Rev. Lett. 101, 057201 (2008).

[28] J. O. Piatek, I. Kovacevic, P. Babkevich, B. Dalla Piazza, S. Neithardt, J. Gavilano, K. W. Krämer, and H. M. Rønnow, Phys. Rev. B 90, 174427 (2014).

[29] M. J. Harris, S. T. Bramwell, D. F. McMorrow, T. Zeiske, and K. W. Godfrey, Phys. Rev. Lett. 79, 2554 (1997).

[30] G. Sala, C. Castelnovo, R. Moessner, S. L. Sondhi, K. Kitagawa, M. Takigawa, R. Higashinaka, and Y. Maeno, Phys. Rev. Lett. 108, 217203 (2012). 